\title{
Utilisation d'un sondage en ligne pour la collecte de renseignements sur l'exposition aux aliments, sous-étude Foodbook, de février à avril 2015
}

\author{
Christine Gardhouse', Matt Hurst ${ }^{1}$, Sujani Sivanantharajah¹, Nadia Ciampa ${ }^{1 \star}$
}

\section{Résumé}

Contexte : Lors des enquêtes sur les éclosions de maladies d'origine alimentaire, la comparaison des fréquences des expositions aux aliments des cas avec celles d'une population témoin peut aider à déterminer les sources alimentaires suspectes. L'Agence de la santé publique du Canada (l'Agence) a mené un sondage en ligne entre février et avril 2015 afin de recueillir des données sur l'exposition aux aliments pendant sept jours à partir d'un échantillon de commodité. La période de l'étude a coïncidé avec un sondage téléphonique au hasard à l'échelle nationale sur l'exposition de la population. Un sous-ensemble de questions sur l'exposition aux aliments provenant du sondage téléphonique a été inclus au sondage en ligne.

Objectif : Les objectifs du sondage en ligne étaient les suivants : 1) décrire la méthodologie du sondage, les répondants et le comportement de réponse; et 2) déterminer si la méthodologie en ligne est une solution de rechange aux sondages téléphoniques en comparant les expositions aux aliments.

Méthodes : Le lien vers le sondage en ligne a été distribué par courrier électronique aux partenaires et aux employés de la santé publique, et a fait l'objet de promotion sur le site Web de l'Agence et dans les médias sociaux.

Résultats : Au total, 2100 sondages ont été réalisés. La majorité des répondants étaient des femmes, âgées de 30 à 39 ans, ayant un revenu et un niveau d'éducation élevés. La proportion de personnes ayant déclaré avoir consommé des produits alimentaires dans le cadre du sondage en ligne était généralement plus élevée que dans le sondage téléphonique, avec une différence moyenne de 6,0\% (IC à $95 \%: 4,2,7,8)$.

Conclusion : Dans une enquête sur une éclosion, le biais de 6,0 \% pourrait rendre plus difficile la détection d'une différence entre le cas et le témoin des expositions aux aliments. Néanmoins, compte tenu de la rapidité des réponses et des dépenses plus faibles des sondages en ligne ainsi que de l'échantillon volontaire, compétent et commode, un biais de $6,0 \%$ est considéré comme suffisamment faible pour être acceptable pour les sondages futurs.
Cette oeuvre est mise à la disposition selon les termes de la licence internationale Creative Commons Attribution 4.0

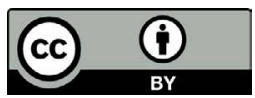

Affiliation

${ }^{1}$ Agence de la santé publique du Canada, Guelph, ON

*Correspondance :

nadia.ciampa@canada.ca

Citation proposée : Gardhouse C, Hurst M, Sivanantharajah S, Ciampa N. L'utilisation d'un sondage en ligne pour la collecte de renseignements sur l'exposition aux aliments, sous-étude Foodbook, de février à avril 2015. Relevé des maladies transmissibles au Canada 2021;47(1):27-34. https://doi.org/10.14745/ccdr.v47i01a05f

Mots-clés : sondage en ligne, échantillon de commodité, expositions aux aliments, maladies d'origine alimentaire, enquêtes sur les éclosions, sondage téléphonique

\section{Introduction}

Lors des enquêtes sur les éclosions de maladies d'origine alimentaire, la comparaison de la fréquence des expositions des cas avec celle d'une population témoin peut aider à déterminer les sources alimentaires suspectes. L'utilisation des données d'exposition de la population existante comme données "témoins " est une solution de rechange utile aux études cas-témoins traditionnelles (1). Généralement, les sondages téléphoniques sont utilisés pour obtenir des données 
sur l'exposition (2), mais comme ils nécessitent beaucoup de ressources et ne sont pas opportuns, il est nécessaire d'explorer d'autres moyens d'obtenir et de mettre à jour les données sur l'exposition.

Ces dernières années, les sondages en ligne ont été de plus en plus utilisés pour collecter des données à des fins de recherche. Les sondages en ligne nécessitent moins de ressources et moins de temps à mettre en œuvre que les méthodes de sondage téléphonique traditionnelles. Ils permettent également une réponse plus rapide et un meilleur accès aux groupes plus difficiles à rejoindre (3-6). D'autre part, l'utilisation de sondages en ligne peut entraîner un biais d'échantillonnage et, si un échantillon fixe n'est pas en place, il peut être difficile de suivre les taux de non-réponse $(7,8)$. Les sondages en ligne pourraient potentiellement être utilisés comme complément aux sondages téléphoniques pour la collecte de données $(3,5)$. Toutefois, l'évaluation de la base d'échantillonnage la plus appropriée à utiliser compte tenu de l'effet sur les résultats est une considération importante.

Étant donné la rapidité d'administration et le faible coût des sondages en ligne, l'Agence de la santé publique du Canada (l'Agence) a cherché à évaluer leur utilisation potentielle pour la collecte de renseignements sur les témoins de l'exposition aux aliments en cas d'éclosion de maladie entérique. Afin de comparer les résultats obtenus par ces deux modes de collecte de données, l'Agence a mené un sondage en ligne, en utilisant un échantillon de commodité, parallèlement à un sondage téléphonique à l'échelle nationale sur l'exposition de la population. II s'agit d'une approche commune pour évaluer l'efficacité (9-12).

Les objectifs de l'étude étaient les suivants : 1) décrire la méthodologie du sondage, les répondants et le comportement de réponse; et 2 ) déterminer si la méthodologie en ligne utilisée, y compris la base d'échantillonnage, est une solution de rechange aux sondages téléphoniques en comparant les expositions aux aliments.

\section{Méthodes}

\section{Sondage téléphonique Foodbook : Base de référence}

Le sondage téléphonique Foodbook à l'échelle nationale, et basée sur une population, a été la référence pour évaluer le sondage en ligne comme méthode de collecte de renseignements sur l'exposition aux aliments. Le sondage téléphonique Foodbook a été mené entre avril 2014 et avril 2015 dans les provinces et territoires canadiens. Le sondage comprenait des questions sur l'alimentation, l'eau et l'exposition des animaux au cours des sept derniers jours. Le sondage téléphonique disposait d'un cadre d'échantillonnage et d'un système de pondération robustes, ce qui en faisait le comparateur idéal. Pour plus de détails sur la méthodologie utilisée, veuillez vous référer au Rapport Foodbook (2).

L'étude Foodbook a été approuvée par le Comité d'éthique de la recherche de Santé Canada et de l'Agence de santé publique du Canada (CER 2013-0025) ainsi que par l'autorité d'éthique de la recherche en santé de Terre-Neuve-et-Labrador (HREB 13,238).

\section{Sondage en ligne Foodbook}

Le sondage en ligne a été conçu comme une sous-étude de l'étude complète Foodbook afin d'évaluer la méthodologie en ligne. Pour faciliter cette comparaison, le calendrier a coïncidé avec le sondage téléphonique Foodbook.

La population étudiée pour le sondage en ligne comprenait les résidents canadiens âgés de 16 ans et plus (ou de 18 ans et plus au Québec), qui n'avaient pas voyagé en dehors de leur province ou territoire de résidence au cours des sept derniers jours. Le sondage en ligne a été lancé le 24 février 2015 et s'est terminé le 10 avril 2015. Les participants ont été recrutés à partir d'un échantillon de commodité.

Tout comme le sondage téléphonique, le sondage en ligne comportait des questions sur la démographie des répondants et l'exposition aux aliments. Les données démographiques recueillies comprennent l'âge, le sexe, la province ou le territoire de résidence, l'éducation et le revenu. Seul un sous-ensemble de questions sur l'exposition aux aliments a été inclus dans le sondage en ligne (soit 168 aliments) afin de réduire le temps de réponse à moins de 10 minutes et de maximiser le taux de réponse. Le sondage en ligne a été créé à l'aide de FluidSurveys et était disponible en anglais et en français.

\section{Technique d'échantillonnage}

Dans une situation d'éclosion, il est essentiel d'obtenir des données " témoins » en temps utile. Un échantillon de commodité est une source utile de ces données. L'équipe de recherche a mis en œuvre diverses méthodes pour promouvoir le sondage et recruter des répondants : promotion initiale du sondage par courriel aux membres internes de l'équipe (environ 40 employés); distribution par courriel d'un genre de bulletin quotidien à tous les employés de l'Agence et de Santé Canada (environ 13800 employés); et courriel aux intervenants provinciaux et territoriaux en santé publique. Le recrutement s'est développé grâce à l'échantillonnage en boule de neige, c'est-à-dire en demandant aux destinataires de transmettre les invitations à d'autres. Le sondage a également fait l'objet d'une promotion sur les médias sociaux de l'Agence (Facebook et Twitter) et des bannières publicitaires sur le site Web de I'Agence. Dans l'ensemble, la base de sondage comprenait des employés de Santé Canada et de l'Agence, des groupes 
d'intervenants en santé publique et en épidémiologie (locaux, provinciaux/territoriaux, fédéraux) et le grand public.

Le sondage visait les résidents canadiens de plus de 16 ans (ou de plus de 18 ans au Québec) qui n'avaient pas voyagé hors de leur province ou territoire de résidence au cours des sept derniers jours. Les réponses par procuration n'étaient pas autorisées. Les critères d'inclusion pour les deux modes de sondage étaient identiques, à l'exception des groupes d'âge et de l'utilisation de procurations.

Le consentement a été demandé aux participants pour la collecte et l'utilisation des données dans le but d'aider les professionnels de la santé publique à enquêter sur les maladies et les éclosions. Les personnes ayant répondu « oui » sont passées à l'étape suivante du sondage.

Certains des éléments non aléatoires du système de collecte de l'échantillon ont été corrigés par l'élaboration d'un poids de sondage. Les pondérations ont été élaborées pour chaque sexe et combinaison de provinces en utilisant les chiffres de population du Recensement de 2011.

\section{Analyse des réponses au sondage, des données démographiques des répondants et de l'exposition aux aliments}

Parmi les analyses effectuées, on compte notamment :

1) l'évaluation des réponses aux sondages selon la méthode de recrutement/référencement; 2 ) la description des caractéristiques démographiques des répondants pour les sondages en ligne et téléphonique; et 3) la comparaison des fréquences d'exposition aux aliments entre les sondages en ligne et téléphonique.

Afin d'évaluer l'incidence des différentes méthodes de recrutement sur les réponses aux sondages en ligne, tous les sondages effectués ont été inclus pour l'analyse initiale sur toute la période de l'étude.

En raison des faibles taux de réponse des personnes âgées de 16 à 19 ans et de 65 ans et plus, ainsi que de celles vivant dans les territoires, et pour garantir une taille d'échantillon suffisante à des fins de comparaison, l'unité d'analyse ou la population échantillon, a été affinée pour des analyses plus approfondies. Le groupe du sondage en ligne Foodbook utilisé dans les analyses ultérieures comprenait les personnes âgées de 20 à 64 ans résidant dans les provinces canadiennes, avec une collecte de données entre le 24 février et le 24 mars 2015.

Le groupe de comparaison du sondage téléphonique Foodbook était composé de personnes du même groupe d'âge et de la même zone géographique, bien qu'il ait eu une période plus longue, du 10 février au 7 avril 2015. Le délai plus long (deux semaines supplémentaires de part et d'autre des dates du groupe du sondage en ligne) a été choisi pour augmenter la taille de l'échantillon et améliorer la détection des différences entre les groupes dans les deux sondages.

Pour évaluer la précision de la méthode du sondage en ligne, les proportions de consommation alimentaire du sondage en ligne Foodbook ont été comparées à celles du sondage téléphonique Foodbook pour la même zone géographique (provinces canadiennes) et le même âge des répondants (âgés de 20 à 64 ans) et pour une fenêtre temporelle similaire.

L'analyse a été réalisée à l'aide du logiciel Stata 13.1 (StataCorp LP, College Station, Texas, États-Unis). Une analyse descriptive a été effectuée pour évaluer la réponse au sondage et les données démographiques des répondants. Les comparaisons de l'exposition aux aliments ont été effectuées en analysant les différences moyennes des proportions d'exposition pondérées dans le groupe du sondage en ligne par rapport au groupe du sondage téléphonique et en testant les résultats pour déterminer la signification statistique des différences observées à l'aide de tests de Wald ajustés. Après que les proportions pondérées aient été calculées à l'aide de svy: proportion, la différence moyenne globale entre ces proportions a été calculée à l'aide de la commande lincom, qui fournit un intervalle de confiance de $95 \%$ et la valeur $p$. L'effet du revenu et du niveau de scolarité sur l'écart moyen a été examiné en post-stratification de ces facteurs.

\section{Résultats}

\section{Taux de réponse au sondage}

Le prélancement du sondage, le 24 février 2015, comprenait un lien sur le site Web de l'Agence (par le biais d'une bannière) et un courriel envoyé à une liste restreinte d'employés. Le 25 février 2015, tous les employés de Santé Canada $(n=12000)$ et de l'Agence $(n=1800)$ ont été informés avec le bulletin interne quotidien de l'organisation envoyé par courriel. Le taux de réponse pour cette méthode a été de 4,6\%. Le 26 février 2015, le sondage a fait l'objet d'une promotion sur les canaux Facebook et Twitter de l'Agence, avec le partage ultérieur des messages. Pendant la période de la campagne sur les médias sociaux (entre le 26 février et le 10 avril 2015), la page Web du sondage Foodbook a été consultée 2777 fois, $33 \%$ des consultations provenant de Facebook ou Twitter. La méthode d'inscription la plus efficace - les invitations par courriel envoyées aux provinces et territoires le 2 mars 2015 et les invitations ultérieures envoyées aux listes de diffusion des groupes de santé publique provinciaux et territoriaux et à d'autres canaux de distribution - a généré plus de 1000 réponses, soit environ $48 \%$ du total des réponses.

À la fin de la campagne (du 24 février au 10 avril 2015), 2612 sondages avaient été soumis. Parmi ceux-ci, les données de 512 répondants ont été exclues de l'analyse parce qu'ils $n$ 'avaient pas donné leur consentement ( $n=35)$, ils ne répondaient pas aux critères d'inclusion $(n=276)$ ou n'avaient 
pas répondu au sondage $(n=201)$. Plus des trois quarts des répondants ( $n=2100 ; 80 \%$ ) ont répondu au sondage dans son intégralité. Parmi ceux qui ont répondu au sondage, la majorité a été renvoyée à celui-ci par des courriels envoyés aux intervenants (qui comprenaient un lien vers le site Web du sondage Foodbook) ( $n=1016 ; 48 \%$ ) puis par le groupe interne de distribution du bulletin d'information quotidien de I'Agence/Santé Canada qui a reçu un lien direct vers le sondage ( $n=639 ; 30 \%)$, Facebook ( $n=326 ; 16 \%$ ), le site Web de I'Agence ( $n=70 ; 3 \%)$, Twitter ( $n=44 ; 2 \%$ ) et autres/inconnus ( $n=5 ;<1 \%$ ) (figure 1 ). En raison de l'approche en boule de neige utilisée pour recruter les répondants, il n'a pas été possible

Figure 1 : Nombre de sondages par date d'achèvement selon la méthode de renvoi au sondage en ligne $(n=2100)$

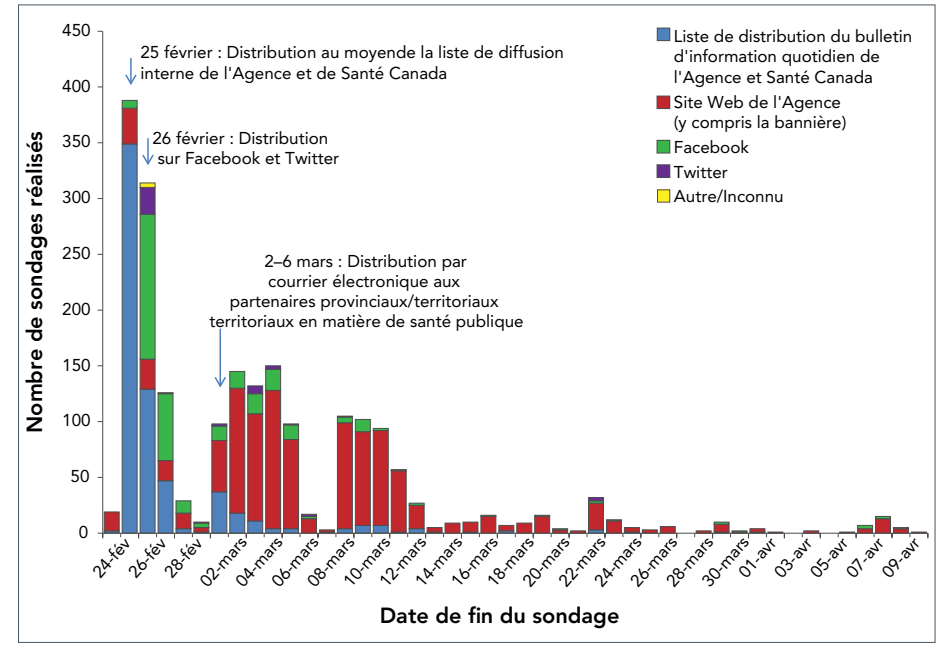

Abréviation : l'Agence, Agence de la santé publique du Canada de saisir toute l'étendue de la promotion et de la distribution du sondage.

Le temps moyen pour répondre au sondage était d'environ 17 minutes (médiane : environ 9 minutes).

\section{Données démographiques des répondants aux sondages en ligne et téléphonique}

Les résultats présentés concernent le " groupe du sondage en ligne ", c'est-à-dire les participants âgés de 20 à 64 ans vivant dans les provinces qui ont répondu au sondage entre le 24 février et le 24 mars 2015 ( $n=1954$ ), et le « groupe du sondage téléphonique » dont le sondage a été effectué entre le 10 février et le 7 avril $2015(n=395)$.

Bien que la répartition des hommes et des femmes participant aux sondages en ligne et téléphonique soit similaire, la répartition des groupes d'âge diffère (tableau 1). La plus grande proportion de participants au groupe du sondage en ligne était constituée des 30 à 39 ans (34,6\%), et au groupe du sondage téléphonique, des 50 à 64 ans $(48,0 \%)$. La répartition géographique des participants était similaire dans toutes les méthodes de sondage. Le groupe du sondage en ligne avait généralement un revenu et un niveau d'éducation plus élevés que le groupe du sondage téléphonique.

Tableau 1 : Données démographiques des participants au groupe du sondage en ligne et téléphonique Foodbook

\begin{tabular}{|c|c|c|c|c|c|}
\hline \multirow[b]{2}{*}{ Caractéristiques } & \multicolumn{2}{|c|}{ Participants en ligne } & \multicolumn{2}{|c|}{ Participants par téléphone } & \multirow[b]{2}{*}{ Valeur $p$} \\
\hline & $\begin{array}{l}\text { Chiffres } \\
\text { bruts (n) }\end{array}$ & $\begin{array}{l}\text { Proportion } \\
\text { pondérée (\%) }\end{array}$ & $\begin{array}{l}\text { Chiffres } \\
\text { bruts (n) }\end{array}$ & $\begin{array}{c}\text { Proportion } \\
\text { pondérée (\%) }\end{array}$ & \\
\hline \multicolumn{6}{|l|}{ Sexe } \\
\hline Hommes & 278 & 49,1 & 150 & 56,5 & 0,155 \\
\hline Femmes & 1676 & 50,9 & 245 & 43,5 & 0,155 \\
\hline \multicolumn{6}{|l|}{ Groupe d'âge } \\
\hline $20-29$ & 369 & 16,5 & 28 & 6,3 & 0,000 \\
\hline $30-39$ & 643 & 34,6 & 43 & 19,5 & 0,000 \\
\hline $40-49$ & 437 & 22,2 & 69 & 26,2 & 0,499 \\
\hline $50-64$ & 505 & 26,8 & 255 & 48,0 & 0,000 \\
\hline \multicolumn{6}{|l|}{ Répondants par province ${ }^{a}$} \\
\hline Colombie-Britannique & 211 & 13,6 & 48 & 9,2 & 0,067 \\
\hline Alberta & 104 & 11,1 & 47 & 9,3 & 0,441 \\
\hline Saskatchewan & 114 & 3,0 & 36 & 3,4 & 0,697 \\
\hline Manitoba & 163 & 3,5 & 33 & 2,1 & 0,037 \\
\hline Ontario & 818 & 37,8 & 75 & 42,4 & 0,429 \\
\hline Québec & 389 & 23,9 & 70 & 26,8 & 0,540 \\
\hline Nouveau-Brunswick & 15 & 2,3 & 25 & 3,0 & 0,543 \\
\hline
\end{tabular}


Tableau 1 : Données démographiques des participants au groupe du sondage en ligne et téléphonique Foodbook (suite)

\begin{tabular}{|c|c|c|c|c|c|}
\hline \multirow[b]{2}{*}{ Caractéristiques } & \multicolumn{2}{|c|}{ Participants en ligne } & \multicolumn{2}{|c|}{ Participants par téléphone } & \multirow[b]{2}{*}{ Valeur $p$} \\
\hline & $\begin{array}{l}\text { Chiffres } \\
\text { bruts (n) }\end{array}$ & $\begin{array}{c}\text { Proportion } \\
\text { pondérée }(\%)\end{array}$ & $\begin{array}{l}\text { Chiffres } \\
\text { bruts (n) }\end{array}$ & $\begin{array}{c}\text { Proportion } \\
\text { pondérée }(\%)\end{array}$ & \\
\hline \multicolumn{6}{|l|}{ Répondants par province (suite) } \\
\hline Nouvelle-Écosse & 37 & 2,8 & 27 & 2,4 & 0,599 \\
\hline Île-du-Prince-Édouard & 56 & 0,4 & 17 & 0,35 & 0,766 \\
\hline Terre-Neuve-et-Labrador & 47 & 1,6 & 17 & 1,0 & 0,224 \\
\hline \multicolumn{6}{|l|}{ Répondants par niveau de revenu (\$) } \\
\hline Moins de $30000 \$$ & 73 & 4,3 & 51 & 8,4 & 0,028 \\
\hline $30000 \$$ ou plus, mais moins de $60000 \$$ & 232 & 12,0 & 106 & 33,5 & 0,000 \\
\hline $60000 \$$ ou plus, mais moins de $80000 \$$ & 280 & 16,7 & 62 & 16,8 & 0,985 \\
\hline $80000 \$$ ou plus & 1150 & 67,0 & 138 & 41,4 & 0,000 \\
\hline \multicolumn{6}{|l|}{ Répondants par niveau d'éducation } \\
\hline Inférieur au diplôme d'études secondaires ou l'équivalent & 8 & 0,3 & 34 & 4,8 & 0,000 \\
\hline $\begin{array}{l}\text { Diplôme d'études secondaires ou une équivalence d'études } \\
\text { secondaires }\end{array}$ & 98 & 5,5 & 90 & 15,9 & 0,002 \\
\hline Certificat ou diplôme professionnel & 59 & 3,6 & 19 & 4,8 & 0,538 \\
\hline $\begin{array}{l}\text { Certificat ou diplôme d'un collège, d'un cégep ou d'un autre } \\
\text { établissement non universitaire }\end{array}$ & 288 & 14,6 & 97 & 25,3 & 0,017 \\
\hline Certificat ou diplôme universitaire inférieur au baccalauréat & 93 & 5,4 & 29 & 10,8 & 0,078 \\
\hline Baccalauréat & 610 & 33,6 & 71 & 28,9 & 0,464 \\
\hline $\begin{array}{l}\text { Certificat ou diplôme d'études universitaires supérieur au } \\
\text { baccalauréat }\end{array}$ & 653 & 36,9 & 39 & 9,5 & 0,000 \\
\hline
\end{tabular}

a Les chiffres bruts et les proportions pondérées pour les territoires n'ont pas été inclus, car les chiffres bruts dans le groupe du sondage en ligne étaient faibles ( $<3$ )

\section{Comparaison des expositions : Résultats du sondage en ligne Foodbook par rapport aux résultats du groupe du sondage téléphonique}

Les expositions aux aliments signalées par les participants au sondage en ligne et téléphonique ont été comparées pour 168 produits alimentaires. La différence entre les proportions pondérées des produits alimentaires variait de 28,6 (épinards) à $-9,4$ (choux-fleurs), avec une médiane de 4,6. Dans l'ensemble, la différence moyenne des proportions de consommation était de 6,0 \% (IC à $95 \%: 4,2 \%, 7,8 \%$ ), des proportions plus élevées étant plus souvent signalées par les répondants au sondage en ligne. Pour les aliments dont plus de $50 \%$ des participants au sondage téléphonique ont déclaré une consommation (i.e. les aliments couramment consommés), la différence moyenne des proportions de consommation entre le sondage en ligne et le sondage téléphonique était de 6,8\%. Le tableau 2 énumère les dix principaux produits alimentaires pour lesquels les différences les plus importantes ont été obtenues entre les deux groupes. Parmi les produits alimentaires présentant les plus grandes différences observées, $50 \%$ étaient des légumes.
Tableau 2 : Les dix principales expositions aux aliments avec les plus grandes différences de proportions pondérées entre les participants au sondage en ligne et au sondage téléphonique

\begin{tabular}{|c|c|c|c|c|c|}
\hline \multirow[b]{2}{*}{$\begin{array}{l}\text { Catégorie } \\
\text { d'aliments }\end{array}$} & \multirow[b]{2}{*}{ Aliment } & \multicolumn{2}{|c|}{ Proportions pondérées } & \multirow{2}{*}{$\begin{array}{l}\text { Différence } \\
\text { entre les } \\
\text { participants } \\
\text { en ligne } \\
\text { et par } \\
\text { téléphone }\end{array}$} & \multirow[b]{2}{*}{$\begin{array}{c}\text { Valeur } \\
p\end{array}$} \\
\hline & & $\begin{array}{l}\text { Participants } \\
\text { en ligne }\end{array}$ & $\begin{array}{c}\text { Participants } \\
\text { par } \\
\text { téléphone }\end{array}$ & & \\
\hline \multicolumn{6}{|c|}{$\begin{array}{l}\text { Les dix principales expositions aux aliments pour lesquelles les résultats des participants } \\
\text { au sondage en ligne étaient plus élevés que les participants par téléphone }\end{array}$} \\
\hline Légumes & Épinards & 56,3 & 27,7 & 28,6 & 0,000 \\
\hline Légumes & $\begin{array}{l}\text { Laitue dans } \\
\text { un sandwich }\end{array}$ & 50,2 & 25,3 & 24,9 & 0,000 \\
\hline $\begin{array}{l}\text { Herbes et } \\
\text { épices }\end{array}$ & $\begin{array}{l}\text { Poudre de } \\
\text { cari }\end{array}$ & 37,9 & 14,4 & 23,5 & 0,000 \\
\hline Légumes & Mesclun & 43,3 & 20,5 & 22,8 & 0,000 \\
\hline Légumes & \begin{tabular}{|l|} 
Tomates \\
cerises ou \\
raisins \\
\end{tabular} & 48,0 & 25,3 & 22,7 & 0,000 \\
\hline Fromage & Mozzarella & 65,2 & 44,1 & 21,1 & 0,000 \\
\hline $\begin{array}{l}\text { Noix et } \\
\text { graines }\end{array}$ & $\begin{array}{l}\text { Beurre } \\
\text { d'arachide }\end{array}$ & 67,2 & 46,7 & 20,5 & 0,000 \\
\hline Bœuf & \begin{tabular}{|l|} 
Hamburgers \\
provenant \\
d'un \\
restaurant \\
ou d'un \\
restaurant- \\
minute \\
\end{tabular} & 31,3 & 11,4 & 19,9 & 0,000 \\
\hline
\end{tabular}


Tableau 2 : Les dix principales expositions aux aliments avec les plus grandes différences de proportions pondérées entre les participants au sondage en ligne et au sondage téléphonique (suite)

\begin{tabular}{|c|c|c|c|c|c|}
\hline \multirow[b]{2}{*}{$\begin{array}{l}\text { Catégorie } \\
\text { d'aliments }\end{array}$} & \multirow[b]{2}{*}{ Aliment } & \multicolumn{2}{|c|}{ Proportions pondérées } & \multirow{2}{*}{$\begin{array}{l}\text { Différence } \\
\text { entre les } \\
\text { participants } \\
\text { en ligne } \\
\text { et par } \\
\text { téléphone }\end{array}$} & \multirow[b]{2}{*}{$\begin{array}{c}\text { Valeur } \\
p\end{array}$} \\
\hline & & $\begin{array}{l}\text { Participants } \\
\text { en ligne }\end{array}$ & $\begin{array}{l}\text { Participants } \\
\text { par } \\
\text { téléphone }\end{array}$ & & \\
\hline Volaille & $\begin{array}{l}\text { Poulet en } \\
\text { morceaux ou } \\
\text { en pièces }\end{array}$ & 81,5 & 62,1 & 19,4 & 0,001 \\
\hline Légumes & $\begin{array}{l}\text { Laitue } \\
\text { emballée }\end{array}$ & 69,8 & 51,3 & 18,5 & 0,001 \\
\hline \multicolumn{6}{|c|}{$\begin{array}{l}\text { Les dix principales expositions aux aliments pour lesquelles les résultats des participants } \\
\text { au sondage en ligne étaient moins élevés que les participants par téléphone }\end{array}$} \\
\hline Légumes & Chou-fleur & 35,7 & 45,1 & $-9,4$ & 0,102 \\
\hline Légumes & Laitue Iceberg & 42,8 & 48,2 & $-5,5$ & 0,345 \\
\hline Légumes & $\begin{array}{l}\text { Germes de } \\
\text { haricot }\end{array}$ & 7,1 & 12,4 & $-5,3$ & 0,090 \\
\hline Légumes & $\begin{array}{l}\text { Choux de } \\
\text { Bruxelles }\end{array}$ & 11,8 & 16,7 & $-5,0$ & 0,171 \\
\hline $\begin{array}{l}\text { Herbes et } \\
\text { épices }\end{array}$ & $\begin{array}{l}\text { Basilic thaï } \\
\text { frais }\end{array}$ & 4,7 & 9,0 & $-4,3$ & 0,182 \\
\hline $\begin{array}{l}\text { Poissons et } \\
\text { fruits de mer }\end{array}$ & $\begin{array}{l}\text { Poissons } \\
\text { (e.g. truites } \\
\text { ou saumons } \\
\text { cuits) }\end{array}$ & 63,7 & 67,3 & $-3,6$ & 0,429 \\
\hline Bœuf & $\begin{array}{l}\text { Bœuf à } \\
\text { ragoût }\end{array}$ & 21,4 & 25,0 & $-3,6$ & 0,562 \\
\hline $\begin{array}{l}\text { Produits } \\
\text { laitiers }\end{array}$ & $\begin{array}{l}\text { Tout produit } \\
\text { laitier cru }\end{array}$ & 6,2 & 9,0 & $-2,8$ & 0,320 \\
\hline Légumes & $\begin{array}{l}\text { Tomates de } \\
\text { serre }\end{array}$ & 42,4 & 45,0 & $-2,6$ & 0,653 \\
\hline Charcuterie & Bologne & 4,5 & 7,1 & $-2,6$ & 0,165 \\
\hline
\end{tabular}

\section{Discussion}

\section{Taux de réponse au sondage}

La rapidité des réponses a varié en fonction de l'approche de recrutement utilisée. Les deux approches qui ont suscité les réponses les plus immédiates ont été la distribution de bulletins d'information internes par courriel aux employés de l'Agence/ Santé Canada et les publications sur Facebook. La plupart des réponses à la distribution du bulletin d'information interne ont eu lieu dans les trois jours, la majorité le jour même de sa réception, ce qui laisse penser qu'il s'agit d'une excellente plateforme pour la collecte de renseignements sensibles au temps. La réponse aux publications sur Facebook a également été rapide, la plupart des sondages ayant été effectués en deux jours. Les invitations à participer au sondage envoyées par courrier électronique aux provinces et territoires, bien que représentant la plus grande proportion des répondants, ont pris environ deux semaines pour produire leur plein effet, probablement en raison de l'approche en boule de neige utilisée.

Nos résultats montrent que la mise en œuvre simultanée de ces trois approches pourrait permettre de réaliser 1600 sondages ou plus en cinq jours ouvrables. Ce serait la ligne la méthode à utiliser pour les sondages sur les éclosions sensibles au temps.

\section{Données démographiques des répondants}

Les résultats pondérés ont indiqué que les répondants de chaque province étaient représentés de manière similaire dans le sondage en ligne et le sondage téléphonique (tableau 1). Cela était attendu, car les pondérations ont été conçues pour corriger les provinces surreprésentées ou sous-représentées. Plus important encore, étant donné que des recherches antérieures ont montré qu'il peut y avoir des disparités dans la répartition des revenus et de l'éducation lorsqu'on utilise des méthodes de sondage en ligne ou téléphonique (13), l'équipe de recherche a comparé les revenus et l'éducation dans les sondages en ligne et téléphonique et a constaté qu'ils étaient différents. Le sondage en ligne avait plus de répondants ayant un revenu et un niveau d'éducation plus élevés que le sondage téléphonique. Ce qui reflète probablement la base de sondage, qui comprend une grande proportion d'employés du gouvernement et de professionnels de la santé publique.

\section{Comparaison des expositions}

Le deuxième objectif de cette étude était de déterminer le rendement d'un sondage en ligne, par rapport à un sondage téléphonique traditionnel, lors de la mesure des proportions d'expositions aux aliments de la population. L'équipe de recherche a évalué la concordance des résultats entre les deux méthodes en comparant les proportions pondérées d'expositions aux aliments du sondage en ligne avec celles du sondage téléphonique.

La proportion de ceux qui consomment les produits alimentaires dans le sondage en ligne tend à être plus élevée que dans le sondage téléphonique. En examinant la différence entre les proportions d'expositions des deux sondages, on a constaté des différences plus ou moins importantes, reflétant la variation d'échantillonnage dans les deux sondages. Les 10 différences les plus importantes, où les proportions étaient plus élevées dans le sondage en ligne que dans le sondage téléphonique, étaient toutes statistiquement significatives; la situation inverse, où les proportions étaient plus faibles dans le sondage en ligne que dans le sondage téléphonique, n'était pas significative. Si la seule variation d'échantillonnage était en jeu, alors la moyenne globale des proportions de consommation ne serait pas différente d'un sondage à l'autre. Toutefois, la différence moyenne est de 6,0\% (IC à $95 \%$ : 4,2 \%, 7,8\%), les proportions plus élevées étant plus souvent rapportées par les répondants au sondage en ligne, ce qui suggère qu'il y a une tendance générale à ce que les répondants au sondage en ligne soient plus susceptibles de répondre qu'ils ont mangé un aliment particulier au cours des sept derniers jours. D'autres travaux ont indiqué que les sondages en ligne, qui utilisent des questions avec deux catégories de réponses demandant des faits plutôt que des opinions, ont des résultats qui correspondent bien au sondage téléphonique (11). 
Il est évident qu'il existe des différences entre les modes de sondage en ligne et téléphonique. Les sondages en ligne sont autoadministrés (plutôt qu'administrés par un intervieweur), et les questions sont présentées visuellement, par écrit (plutôt que posées verbalement), deux facteurs qui peuvent avoir un effet sur les résultats. Aussi, comme l'ont constaté Potoglou et al. (5), il existe potentiellement une plus grande volonté d'être honnête étant donné l'anonymat d'un sondage en ligne. Cependant, il est également possible que, puisqu'un sondage en ligne est autoadministré, la responsabilité pourrait être réduite et la facilité de répondre « oui » pourrait être accrue. La fatigue du répondant peut également avoir un effet sur le comportement du participant lorsqu'il répond, bien que cela puisse être un facteur à la fois pour le sondage téléphonique et le sondage en ligne, selon la durée. Tous ces facteurs peuvent avoir contribué aux différences moyennes globales entre les modes de sondage.

Une autre explication possible de ce biais est l'effet de distribution résultant du fait qu'un plus grand nombre de personnes ayant des revenus élevés ou un niveau d'études supérieur ont répondu au sondage en ligne. Ce point a été approfondi en comparant les résultats du groupe du sondage en ligne après stratification par revenu et par niveau d'études aux mêmes résultats post-stratification du groupe du sondage téléphonique. Aucun modèle ou tendance discernable n'a été trouvé dans les types d'aliments consommés, que ce soit pour le revenu ou l'éducation. De plus, le biais global positif des résultats du sondage en ligne était toujours présent.

\section{Utilisation potentielle dans les enquêtes sur les éclosions}

Le biais de 6,0 \% signifie que les proportions calculées à partir d'un sondage en ligne similaire seraient plus importantes, en moyenne, que celles d'un sondage téléphonique, ce qui se traduirait par un plus grand dénominateur dans un rapport de cotes cas-témoins, d'où un rapport de cotes global plus faible. La détection d'une différence entre le cas et le témoin des proportions d'expositions alimentaire pourrait ainsi être plus difficile. Pour les aliments les plus couramment consommés (i.e. ceux dont la consommation dépasse $50 \%$ selon les résultats du sondage téléphonique), la différence n'augmente pas beaucoup (i.e. 6,8 \% par rapport à 6,0 \%). Bien que les aliments couramment consommés soient déjà plus difficiles à détecter comme sources potentielles ou facteurs de risque (dans un rapport de cotes), le biais de 6,8 \% (par rapport à 6,0\%) n'est pas considéré comme important et n'aurait pas d'incidence sur l'analyse dans la plupart des situations.

\section{Limites}

Malgré le succès global du sondage en ligne Foodbook relativement à la réponse et la comparabilité générale des proportions d'expositions avec celles du sondage téléphonique, la stratégie d'échantillonnage de commodité utilisée se prêtait à un biais potentiel, certaines populations démographiques (i.e. les femmes, les personnes à revenu élevé et les personnes ayant un niveau d'éducation élevé) étant surreprésentées. De plus, les méthodes de recrutement du sondage en ligne n'ont pas permis d'obtenir suffisamment de réponses des territoires et des personnes de moins de 20 ans ou de ou 65 ans et plus. II est également important de noter que, si l'on considère les limites et la pertinence du sondage en ligne par rapport au sondage téléphonique, l'utilisation d'un sondage téléphonique présente également des inconvénients, car il s'agit d'un mode de collecte de données de plus en plus obsolète et exigeant relativement aux ressources.

\section{Conclusion}

Dans l'ensemble, compte tenu de la rapidité et de la réduction des ressources nécessaires pour le sondage en ligne Foodbook utilisant une méthode d'échantillonnage de commodité, ainsi que de l'échantillon volontaire, compétent et commode, un biais de $6,0 \%$ est considéré comme suffisamment faible pour être acceptable pour les sondages où la rapidité est une exigence clé. De plus, étant donné la popularité croissante et la préférence pour l'utilisation de sondages en ligne comme outil de collecte de données, qui devrait continuer à se développer, I'utilisation du mode de collecte de données en ligne, de concert avec d'autres techniques qui améliorent la représentativité de la base de sondage, vaut également la peine d'être explorée pour les futurs sondages qui cherchent à être la nouvelle référence.

\section{Déclaration des auteurs}

C. G. - Conceptualisation et réalisation de l'étude, conceptualisation, rédaction ou révision du document M. H. - Conceptualisation, analyse et interprétation des données, rédaction ou révision du document

S. S. - Conceptualisation, analyse et interprétation des données, rédaction ou révision du document

N. C. - Aide à la mise en œuvre de l'étude, à la

conceptualisation, à la rédaction ou à la révision du document

\section{Intérêts concurrents}

Aucun.

\section{Remerciements}

Les auteurs remercient la Division de la surveillance des maladies d'origine alimentaire et de la résistance aux antimicrobiens (Centre des maladies infectieuses d'origine alimentaire, environnementale et zoonotique, Agence de la santé publique du Canada), ainsi que les épidémiologistes provinciaux et territoriaux pour leur aide dans le cadre du projet Foodbook. Les auteurs remercient également L. Guarna et l'équipe des communications numériques (Direction générale des communications et des affaires publiques, Santé Canada) pour leur aide en ce qui concerne les communications et les aspects du sondage en ligne liés au site Web. Enfin, les auteurs 
remercient J. Cutler, A. Kerr et S. Connors pour leur participation à l'analyse et à l'interprétation des résultats du projet de sous-étude du sondage en ligne.

\section{Financement}

Ce travail a été soutenu par l'Agence de santé publique du Canada, dans le cadre du projet d'étude global Foodbook.

\section{Références}

1. Jervis $R H$, Booth $H$, Cronquist $A B$, Rounds J, Alden NB, Hedberg CW. Moving away from population-based case-control studies during outbreak investigations. J Food Prot 2019 Aug;82(8):1412-6. DOI PubMed

2. Centre des maladies infectieuses d'origine alimentaire, environnementale et zoonotique. Direction Générale de la Prévention et Contrôle des Maladies Infectieuses. Rapport foodbook. Ottawa (ON) : Agence de la santé publique du Canada; 2015 (modifié 2016-05-02; accédé 2020-1222). https://www.canada.ca/fr/sante-publique/services/ publications/aliments-et-nutrition/rapport-foodbook.html

3. Kaplowitz MD, Hadlock TD, Levine R. A comparison of web and mail survey response rates. Public Opin $\mathrm{Q}$ 2004;68(1):94-101. DOI

4. Messer BL, Dillman DA. Surveying the general public over the internet using address-based sampling and mail contact procedures. Public Opin Q 2011;75(3):429-57. DOI

5. Potoglou D, Kanaroglou PS, Robinson N. Evidence on the comparison of telephone and internet surveys for respondent recruitment. Open Transplant J 2012;6:11-22. $\mathrm{DOI}$
6. Wright KB. Researching internet-based populations: advantages and disadvantages of online survey research, online questionnaire authoring software packages, and web survey services. J Comput Mediat Commun 2005;10(3). DOI

7. Berrens RP, Bohara AK, Jenkins-Smith $H$, Silva $C$, Weimer DL. The advent of internet surveys for political research: a comparison of telephone and internet samples. Polit Anal 2003;11(1):1-22. DOI

8. Lindhjem $\mathrm{H}, \mathrm{Navrud} \mathrm{S}$. Are internet surveys an alternative to face-to-face interviews in contingent valuation? Ecol Econ $2011 ; 70(9): 1628-37$. DOI

9. Fricker S, Galesic M, Tourangeau R, Yan T. An experimental comparison of web and telephone surveys. Public Opin $\mathrm{Q}$ 2005;69(3):370-92. DOI

10. Roster CA, Rogers RD, Albaum G, Klein D. A comparison of response characteristics from web and telephone surveys. Int J Mark Res 2004;46(3):359-73. DOI

11. Schonlau M, Zapert K, Simon LP, Sanstad KH, Marcus SM, Adams J, Spranca M, Kan H, Turner R, Berry SH. A comparison between responses from a propensity-weighted web survey and an identical RDD survey. Soc Sci Comput Rev 2003;21(10):1-11. DOI

12. Smith B, Smith TC, Gray GC, Ryan MA; Millennium Cohort Study Team. When epidemiology meets the Internet: Web-based surveys in the Millennium Cohort Study. Am J Epidemiol 2007;166(11):1345-54. DOl PubMed

13. Dillman DA, Smyth JD, Christian LM. Internet, phone, mail, and mixed-mode surveys. 4th ed. Hoboken (NJ): John Wiley \& Sons, Inc.; 2014. 